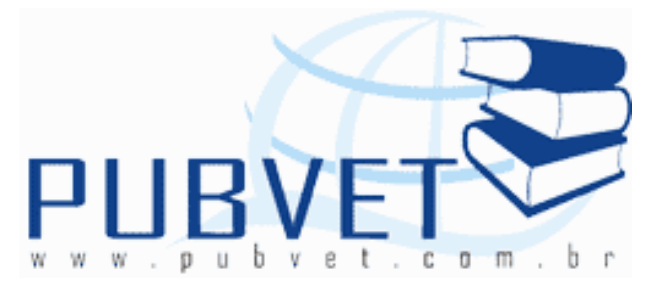

PUBVET, Publicações em Medicina Veterinária e Zootecnia.

\title{
Estudo radiográfico contrastado do tempo de trânsito gastrintestinal em Caracara plancus
}

\footnotetext{
André Luiz Quagliatto Santos ${ }^{3}$, Juliana Macedo Magnino Silva ${ }^{1}$, Arthur Paulino Sanzo Kaminishi ${ }^{1}$, Simone Borges Salgueiro De Simone ${ }^{1}$, Jerônimo Luiz Cardoso $^{1}$, Lorena Tannús Menezes ${ }^{1}$, Heloísa Castro Pereira ${ }^{2}$

${ }^{1}$ Mestrando em Ciências Veterinárias - UFU

${ }^{2}$ Residente Médico Veterinário Residente Hospital Veterinário - UFU

${ }^{3}$ Docente do Programa de Pós-graduação em Ciências Veterinárias - UFU
}

\section{Resumo}

O caracará é uma ave com reconhecido papel ecológico de controle de populações, principalmente de pequenas aves e mamíferos. Em aves de cativeiro, radiografias contrastadas tem sido utilizadas para observar estruturas assim como estudar o tempo de trânsito alimentar. Com o objetivo de determinar o tempo de trânsito gastrintestinal em caracarás, utilizou-se radiografia contrastada com solução de sulfato de bário a $100 \%$ na dose de 5 $\mathrm{ml} / \mathrm{Kg}$ em seis aves. As tomadas radiográficas foram feitas na posição láterolateral, nos tempos em minutos: zero, dez, 25, 40, 55, 70, 85, 100 e 130, continuando de 30 em 30 minutos até completa eliminação do contraste. Foi aplicada estatística simples com obtenção de médias e desvio padrão dos resultados encontrados. A técnica radiográfica utilizada foi eficaz e o tempo 
médio de permanência do sulfato de bário em Caracara plancus foi de $670 \pm$ 80,5 minutos.

Palavras-chave: Caracará, sulfato de bário, trato gastrintestinal, radiografia.

\title{
Evaluation of gastrointestinal tract transit times using barium sulfate suspension in Caracara plancus
}

\begin{abstract}
Crested Caracara is an opportunist feeder, eating some variety of food. In pet and captive avian species, barium sulfate suspension has been used to assess gastrointestinal structure, as well to observe gastrointestinal transit. The purpose of this study was to determine gastrointestinal transit times in Caracara plancus using $5 \mathrm{mg} / \mathrm{Kg}$ of a $100 \%$ barium sulfate suspension. Wholebody latero-lateral view radiographs were taken at zero, ten, 25, 40, 55, 70, 85,100 e 130 minutes. Barium sulfate suspension can be a useful tool in characterizing gastrointestinal function radiographically in Caracara plancus. Complete gastrointestinal emptiness ocurred at $670 \pm 80.50$ minutes.

Keywords: Crested Caracara, barium sulfate, gastrointestinal tract, radiography.
\end{abstract}

\section{Introdução}

O caracará é uma ave onívora e sua alimentação constitui basicamente de frutas, detritos, cadáveres, aves vivas, anelídeos e anfíbios (Hertel, 1995). Animais onívoros possuem a digestão enzimática, semelhante à de carnívoros.

Aves de rapina podem ser acometidas por variadas obstruções, neoplasias, presença de corpos estranhos, impactações e infecções fúngicas em seu trato gastrintestinal (Pinto, 2007). A utilização de exames radiográficos mostrou-se eficiente para diagnóstico e terapêutica de impactações 
SANTOS, A.L.Q. et al. Estudo radiográfico contrastado do tempo de trânsito gastrintestinal em Caracara plancus. PUBVET, Londrina, V. 5, N. 13, Ed. 160, Art. 1084, 2011.

(Dumonceaux et al., 1994), obstruções (Das et al., 2008), neoplasias e hérnias no trato digestório (Grimm et al., 1991; Rübel, 1991).

A utilização do sulfato de bário como contraste radiográfico é a melhor alternativa para fornecer informações sobre a morfologia, obstrução e patologias do trato gastrintestinal (Chen, 1999). Em aves de cativeiro, este contraste tem sido utilizado para observar estruturas assim como determinar o tempo de transito alimentar (Bloch, 2010).

O conhecimento do tempo de TGI (trânsito gastrintestinal) pode auxiliar na formulação de dietas condizentes com a fisiologia digestiva de aves em cativeiro e no diagnóstico e tratamento de patologias deste sistema. Assim, objetivou-se determinar o tempo de trânsito gastrintestinal em Caracara plancus utilizando radiografia contrastada com solução de sulfato de bário a $100 \%$.

\section{Material e Métodos}

O experimento foi conduzido no Laboratório de Ensino e Pesquisa em Animais Silvestres (LAPAS) e Hospital Veterinário da Universidade Federal de Uberlândia, após aprovação do Comitê de Ética na Utilização de Animais (CEUA) da Universidade Federal de Uberlândia (UFU) com o registro de no. $103 / 10$.

As aves passaram por um exame físico de acordo com Werther (2008) e foram alojadas em viveiros telados, com acesso a luz solar e alimentadas com três pintos de um a dez dias de idade, oferecidos diariamente para cada animal. Foram utilizados seis espécimes de Caracara plancus adultos, hígidos, provenientes da entrega voluntária pela Polícia Ambiental e IBAMA ao Ambulatório de Animais Silvestres do Hospital Veterinário da Universidade Federal de Uberlândia.

O contraste utilizado foi o sulfato de bário a $100 \%$ (Bariogel $($ ) ) em suspensão, na dose de $5 \mathrm{ml} / \mathrm{Kg}$ (Delafiori et al, 2009), administrado via oral 
SANTOS, A.L.Q. et al. Estudo radiográfico contrastado do tempo de trânsito gastrintestinal em Caracara plancus. PUBVET, Londrina, V. 5, N. 13, Ed. 160, Art. 1084, 2011.

por meio de uma sonda esofágica descartável diretamente no papo. Os caracarás foram alimentados 15 horas antes da administração do contraste.

Após ingestão do sulfato de bário, as aves foram radiografadas na posição látero-lateral no aparelho de raio X Triplunix $800 \mathrm{~mA}$ regulado para 52 $\mathrm{Kv}, 50 \mathrm{~mA}$ e tempo de exposição de 0,032 segundos. Utilizou-se filme radiográfico médico (Fujifilm $®$ ) de $30 \times 40 \mathrm{~cm}$. As tomadas radiográficas foram feitas nos tempos em minutos: zero, dez, 25, 40, 55, 70, 85, 100 e 130, continuando de 30 em 30 minutos até completa eliminação do contraste.

Foi aplicada estatística descritiva simples com obtenção de médias e desvio padrão dos resultados após análise das radiografias.

\section{Resultados}

A seqüência radiográfica realizada após administração do material contrastante, possibilitou a visualização e registro do tempo de preenchimento e esvaziamento do contraste nas estruturas do tubo digestório. Todas as aves sobreviveram ao experimento.

Imediatamente após a ingestão do contraste, houve preenchimento do ventrículo e proventrículo em todas as aves (tabela 1 ). O tempo total de permanência da suspensão de sulfato de bário no trato digestório de Caracara plancus foi de $670 \pm 80,50$ minutos (Tabela 2).

Tabela 1: Tempo de preenchimento em minutos da suspensão de sulfato de bário a $100 \%$ dos segmentos do tubo digestivo dos exemplares 1, 2, 3, 4, 5 e 6 de Caracara plancus. Uberlândia, 2010.

\begin{tabular}{c|ccccc|} 
& \multicolumn{5}{|c|}{ PREENCHIMENTO } \\
\hline Animal & Proventriculo & Ventriculo & Duodeno & Jejuno/íleo & Colorreto \\
\hline 1 & $*$ & $*$ & $*$ & $*$ & 70 \\
2 & $*$ & $*$ & $*$ & $*$ & 25 \\
3 & $*$ & $*$ & 10 & 10 & 70 \\
4 & $*$ & $*$ & $*$ & 10 & 25 \\
5 & $*$ & $*$ & 10 & 25 & 40 \\
6 & $*$ & $*$ & 25 & 25 & 130 \\
\hline
\end{tabular}


SANTOS, A.L.Q. et al. Estudo radiográfico contrastado do tempo de trânsito gastrintestinal em Caracara plancus. PUBVET, Londrina, V. 5, N. 13, Ed. 160, Art. 1084, 2011.

Tabela 2: Tempo de esvaziamento em minutos da suspensão de sulfato de bário a $100 \%$ dos segmentos do tubo digestivo dos exemplares 1, 2, 3, 4, 5 e 6 de Caracará plancus. Uberlândia, 2010.

\begin{tabular}{c|ccccc|} 
& \multicolumn{5}{|c|}{ ESVAZIAMENTO } \\
\hline Animal & Proventriculo & Ventriculo & Duodeno & Jejuno/íleo & Colorreto \\
\hline 1 & 640 & 640 & 640 & 0 & 700 \\
2 & 520 & 520 & 610 & 730 & 730 \\
3 & 700 & 700 & 700 & 700 & 730 \\
4 & 340 & 340 & 340 & 400 & 520 \\
5 & 610 & 610 & 610 & 640 & 640 \\
6 & 520 & 520 & 520 & 640 & 700 \\
\hline
\end{tabular}

\section{Discussão}

A posição látero-lateral possibilitou a visualização do proventrículo, ventrículo, duodeno, jejuno, íleo e colorreto (Figura 1), corroborando com VinkNooteboom et al. (2003). Já Bloch et al. (2010) e Delafiori et al. (2009) radiografaram pombos domésticos (Columba livia) e tucanos de bico verde (Ramphastos dicolorus) respectivamente na posição ventro dorsal. Em nenhuma radiografia foi possível visualizar os cecos, pois, de acordo com Mitchell (1901), os cecos em carcarás são vestigiais.

Para a visualização da estruturas do trato digestório e mensuração do tempo de passagem do alimento, a suspensão de sulfato de bário foi satisfatória tanto para a pesquisa em questão, quanto para os estudos de Wencke \& Kirberger (2003), Vink-Noteboom (2003), Pinto (2007) e Bloch (2010).

Franzo et al. (2007) relataram que o comprimento do intestino delgado de caracarás, especialmente o duodeno é maior quando comparado com o de outras espécies de aves. Através das radiografias, observou-se a grande extensão do intestino delgado, o que pode justificar o longo tempo de passagem do contraste por esta estrutura. Segundo Hill (1976), Sturkie (1986) e Banks (1992), um longo período de digestão é necessário para que ocorra a absorção de nutrientes com maior eficiência.

O preenchimento dos estômagos em Caracara plancus ocorreu imediatamente após a administração do contraste, e o esvaziamento foi de $555 \pm 126,4$ 
SANTOS, A.L.Q. et al. Estudo radiográfico contrastado do tempo de trânsito gastrintestinal em Caracara plancus. PUBVET, Londrina, V. 5, N. 13, Ed. 160, Art. 1084, 2011.

minutos. Em avestruzes, o preenchimento também foi imediato à aplicação, e o esvaziamento de no máximo 300 minutos. A longa permanência do alimento nos estômagos ocorre para que haja melhor trituração, já que aves não possuem capacidade de mastigação (Wencke \& Kirberger, 2003).

O tempo de TGI em caracarás foi de $670 \pm 80,5$ minutos. Em estudos semelhantes, foram relatados tempos de passagem do contrate de 300 minutos em papagaios (Vink-Nooteboom et al., 2003), 360 minutos em falcões (Mcmillan, 1994), 960 minutos em avestruzes (Wencke \& Kirberger, 2003) e 1440 minutos em pombos (Bloch et al., 2010). Periquitos australianos, faisões, (Mcmillan, 1994) e tucanos de bico verde (Delafiori et al., 2009) tiveram o tempo de TGI variando entre 120 e 240 minutos. 
SANTOS, A.L.Q. et al. Estudo radiográfico contrastado do tempo de trânsito gastrintestinal em Caracara plancus. PUBVET, Londrina, V. 5, N. 13, Ed. 160, Art. 1084, 2011.

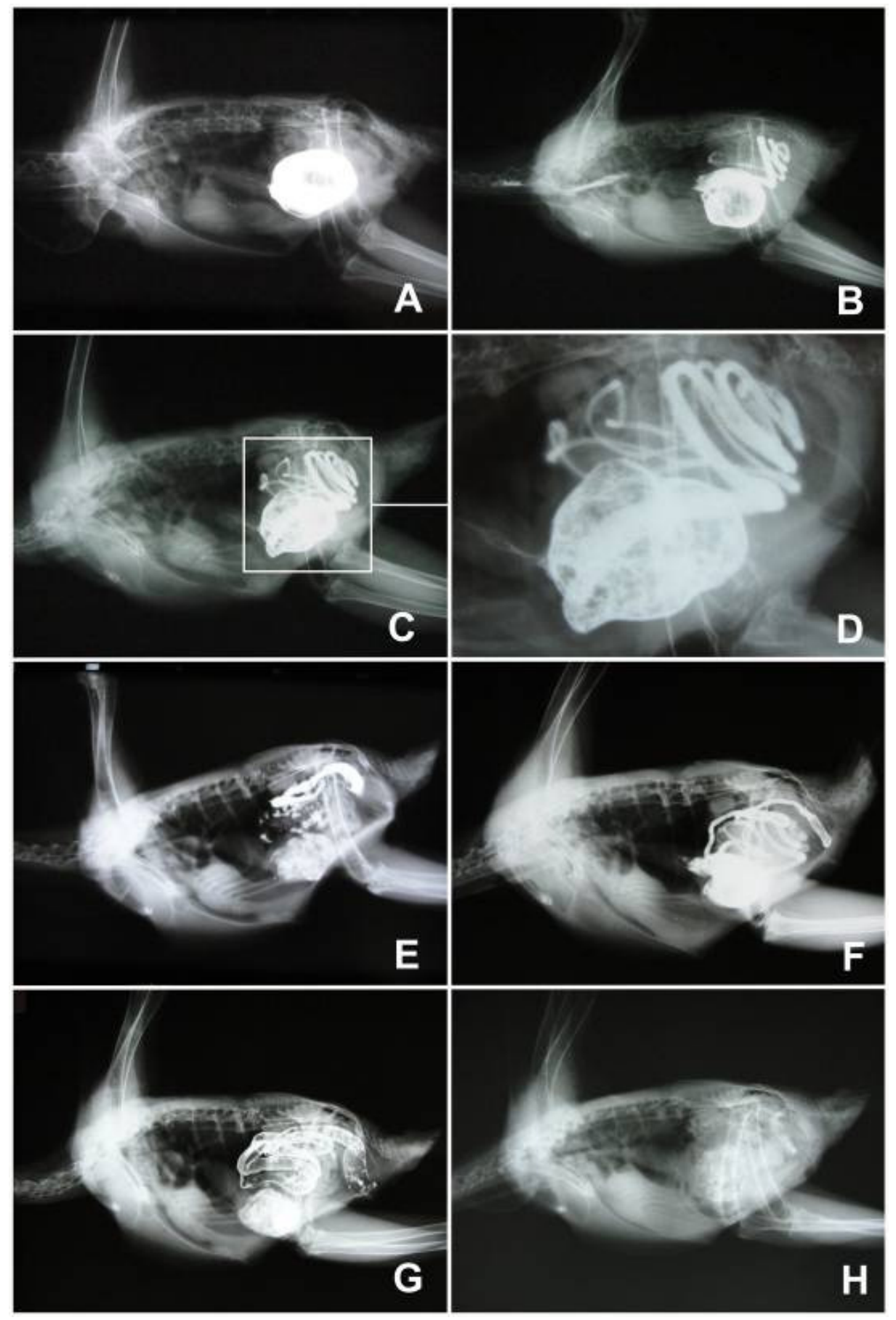

Figura 1: Fotografias da sequência de preenchimento e esvaziamento do trato gastrintestinal de Caracara plancus. A, preenchimento imediato do rpoventrículo e ventrículo; B, preenchimento do duodeno e porção inicial do jejuno-íleo; C, preenchimento completo do jejuno-íleo; D, aumento da fotografia C para observação do preenchimento do jejuno-íleo e duodeno; E, esvaziamento do proventrículo e ventrículo e preenchimento da porção inicial do colo-reto; F, preenchimento de todas as estruturas do trato digestório; G, esvaziamento do jejuno-íleo e colo-reto, explicitando a impregnação de contraste na cloaca; $\mathbf{H}$, eliminação total do contraste. 
SANTOS, A.L.Q. et al. Estudo radiográfico contrastado do tempo de trânsito gastrintestinal em Caracara plancus. PUBVET, Londrina, V. 5, N. 13, Ed. 160, Art. 1084, 2011.

\section{Conclusão}

A técnica de radiografia contrastada com suspensão de sulfato de bário a $100 \%$ foi eficaz para observar as estruturas e determinar o tempo de trânsito do trato gastrintestinal em caracarás (Caracara plancus). O tempo médio de permanência do sulfato de bário foi de $670 \pm 80,5$ minutos.

\section{Referências}

BANKS, W. J. Histologia veterinária aplicada. 2a ed. Manole, São Paulo, 1992. p.629.

BLOCH, R. A.; CRONIN, K.; HOOVER, J. P.; PECHMAN, R. D.; PAYTON, M. E. Evaluation of gastrointestinal tract transit times using barium-impregnated polyethylene spheres and barium sulfate suspension in a domestic pigeon (Columbia livia) model. Journal of Avian Medicine and Surgery, v.24, n.1, p.1-8, 2010.

CHEN, J. Reassessment of barium radiographic examination in diagnosing gastrointestinal diseases. World Journal of Gastroenterology, v.5, n.5, p.383-387, 1999.

DAS, B. C.; AZIZUNNESA, B. C.; SUTRADHAR, M. O.; FARUK, M. O. Oesophageal impaction in an indigenous goose. Bangladesh Journal of Veterinary Medicine. v. 6, n. 2, p. 231-232, 2008.

DELAFIORI, D. M., CAVALCANTE, M. K., GUEDES, P. M., TAKAESU, A. Y., DEELAFIORI, R. E., QUAGLIA NETO, F. \& GOMES, M. S. Estudo radiográfico contrastado do sistema digestório de tucanos de bico verde (Ramphastos dicolorus). Nosso Clínico. v.12, n. 69, p. 39-41, 2009.

DUMONCEAUX, G., HARRISON, G. J., RITCHIE, B. W., HARRISON, G. J. \& HARRISON, L. R. Avian medicine principles and application. Wingers Publishing, Lake Worth, 1994. 1384p.

FRANZO, V. S., ARTONI, S. M. B., VULCANI, V. A. S., SAGULA, A. \& MORAES, C. Análise biométrica do intestino do carcará (Polyborus plancus, Miller, 1777). Biotemas, v. 20, p. 83-8, 2007.

GRIMM F., RÜBEL G. A., ISENBÜGEL E. \& WOLVEKAMP P. Vögel: Kontrastmittelstudien zur Darstellung des Verdauungstraktes. In: Atlas der Röntgendiagnostik bei Heimtieren. 1. Aufl., Schlütersche, Hannover, S., 1991. p.126-137.

HERTEL, F. Ecomorphological indicators of feeding behavior in recent and fosil raptors. The Auk. v.102, n.4, p.890-903, 1995.

HILL, K. J. The anatomy and general physiology of the alimentary tract. In: Simposium of digestion in the fowl, 1976, Anais... Brithish Poultry Science, Edinburgh, Scotland, p.3-24, 1976.

MCMILLAN, M. C. Imaging Techniques. In: RITCHIE B. W, HARRISON G. J, HARRISON L. Avian Medicine, Principles and Application. Lake Worth, Florida: Wingers Publishing Inc., 1994. p.256-259. 
SANTOS, A.L.Q. et al. Estudo radiográfico contrastado do tempo de trânsito gastrintestinal em Caracara plancus. PUBVET, Londrina, V. 5, N. 13, Ed. 160, Art. 1084, 2011.

MITCHELL, P. C. On the intestinal tract of birds, with remarks on the valuation and nomenclature of zoological characters. Transamerica Society of London, v. 8 p.173-275, 1901.

PINTO A. C. B. C. Radiologia In: CUBAS Z. S.; SILVA, J. C. R.; CATÃO-DIAS J. L. Tratado de Animais Selvagens, $1^{\text {a }}$ Ed, ROCA, 2007. p.896-919.

RÜBEL G. A. VÖGEL. In: RÜBEL G.A., ISENBÜGEL E., WOLVEKAMP P. Atlas der Röntgendiagnostik bei Heimtieren. Schlütersche, Hannover, S., p.76-83, 1991.

STURKIE, P. D. Avian Physiology. 4th ed. Springer-Verlag, New York, USA, 1986. 675p.

VINK-NOTEBOOM, M.; LUMEIJ, J. T.; WOLVVEKAMP, W. T. C. Radiography and imageintensified fluoroscopy of barium passage through the gastrointestinal tract in six healthy amazon parrots (Amazona aestiva). Veterinary Radiology \& Ultrasound,v.44, n.1, p.43-48, 2003.

WENCKE, M. W. \& KIRBERGER, R. M. Radiographic gastrointestinal contrast study in the ostrich (Struthio camelus). Veterinary Radiology \& Ultrasound, v. 44, n. 5, p. 546-552, 2003.

WERTHER, K. Semiologia de Animais Silvestres. In: FEITOSA, F. L. Semiologia - A Arte do Diagnóstico. 2 ed. Roca, São Paulo, 2008. p.655-718. 